Document downloaded from:

http://hdl.handle.net/10251/98275

This paper must be cited as:

Morales Torres, A.; Escuder Bueno, I.; Altarejos García, L.; Serrano Lombillo, AJ. (2016). Building fragility curves of sliding failure of concrete gravity dams integrating natural and epistemic uncertainties. Engineering Structures. 125:227-235.

doi:10.1016/j.engstruct.2016.07.006

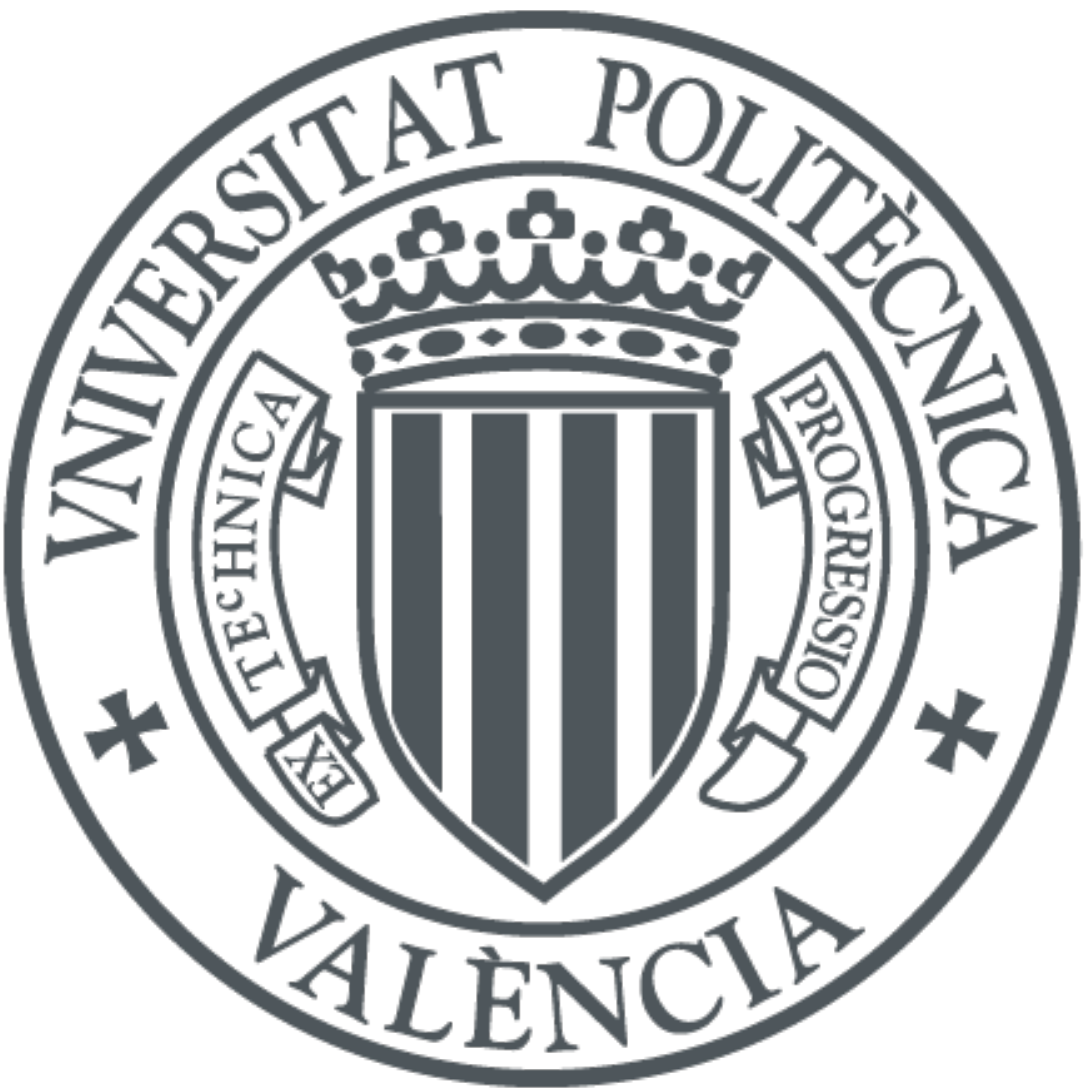

The final publication is available at

https://doi.org/10.1016/j.engstruct.2016.07.006

Copyright Elsevier

Additional Information 


\title{
Building fragility curves of sliding failure of concrete gravity dams integrating natural and epistemic uncertainties
}

\author{
Adrián Morales-Torres ${ }^{\mathrm{a}, *}$, Ignacio Escuder-Bueno ${ }^{\mathrm{b}}$, Luis Altarejos-García ${ }^{\mathrm{c}}$, \\ Armando Serrano-Lombillo ${ }^{b}$ \\ ${ }^{a}$ iPresas Risk Analysis, Avenida del Puerto 180, $1^{\circ}$-B. 46023 Valencia - Spain, \\ Tel.: +34637211465 \\ ${ }^{b}$ Universitat Politècnica de València. Research Institute of Water and Environmental \\ Engineering. Camino de Vera, $s / n$ 46022 Valencia - Spain \\ ${ }^{c}$ Universidad Politécnica de Cartagena. Ud. Predepartamental de Ingeniería Civil. Paseo \\ de Alfonso XIII, 5230203 Cartagena - Spain
}

\begin{abstract}
In the majority of engineering problems, two kinds of uncertainty are generally considered: natural uncertainty, resulting from the inherent variability in natural processes, and epistemic uncertainty, linked to lack of knowledge. When performing a quantitative risk analysis, considering both types of uncertainty separately before integrating them when performing risk calculations, allows a better understanding on how both types of uncertainty influence risk results.

The main purpose of this paper is presenting a consistent procedure to perform fragility analysis for dams in order to identify and track natural and epistemic uncertainty separately. This procedure is particularized for the sliding failure mode of concrete gravity dams, due to its importance. The resulting fragility curves provides a valuable input to quantitative risk models in order to compare the effect of risk reduction and uncertainty reduction investments.
\end{abstract}

The proposed procedure combines the concepts of the Electrical Power

\footnotetext{
*Corresponding author

Email addresses: adrian.morales@ipresas.com (Adrián Morales-Torres), iescuder@hma.upv .es (Ignacio Escuder-Bueno), luis.altarejos@upct .es (Luis Altarejos-García), arserlom@alumni.upv.es (Armando Serrano-Lombillo)
} 
Research Institute (EPRI) guidelines to develop fragility curves for the nuclear industry with existing reliability techniques for computing fragility curves in the context of concrete dams engineering. The procedure has been applied to a dam to illustrate how it can be used in a real case in such a manner that fragility curves are obtained integrating natural and epistemic uncertainties without losing track of their separate contribution to risk results.

Keywords: Fragility analysis, Concrete dams, Sliding failure mode, Natural and epistemic uncertainty, Quantitative risk analysis

\section{Introduction}

Engineering tools such as risk analysis can be useful to inform decisions regarding dam safety governance [1]. Risk assessment tools and techniques are routinely used by several industries $[2,3,4,5,6]$. Benefits from the risk analysis approach are recognized even when limited data are available as risk assessment helps engineers to understand uncertainties in a project, and provides a logical process of identifying hazards, evaluate the severity of each hazard, and assess the effectiveness of risk reduction measures [7].

However, the contextual information provided above is way more complex than it may sound, veiling lots of theoretical and practical difficulties. Many of these difficulties are related to how uncertainties are explicitly considered today (in the context of risk analysis), in contrast to the more traditional implicit treatment (in the context of state-of-the-art dam safety practice).

With regard to uncertainties present in the analysis of the future behavior of a constructed facility, whose analysis should play an important role in the dam safety evaluation, many authors have identified two distinctive categories or sources $[8,9,10,11,4]$ as shown in figure 1 :

- Natural uncertainty or randomness: produced by the inherent variability in the natural processes. It includes the variability along time of phenomena that take place in a precise point of the space (temporal variability) or the variability across the space of phenomena that take place in different points but simultaneously (spatial variability).

An example of this kind of uncertainty is the variability of the loads that the structure has to withstand, for instance, the variability in the 
potential intensity of earthquakes. Another example is the strength's variability of the foundation where the structure stands. This type of uncertainty, sometimes also called aleatoric uncertainty, cannot be reduced, though it can be estimated.

- Epistemic uncertainty: resulting from lack of knowledge or information about the analyzed system. This uncertainty can be divided in two categories: uncertainty of the model and uncertainty of the parameters. The uncertainty of the model refers to the ignorance of the extent to which a model reproduces reality faithfully. It reflects the incapacity of representing reality or of identifying the best model to do it. The uncertainty in the parameters arises from the restricted capacity to estimate them in an adequate manner from a limited number of data from tests or calibration, including measurement errors (related to the meter or the operator), survey error and also from the inherent limitations of the statistical techniques used in the estimation of the parameters. The more knowledge is available about a structure, the more this type of uncertainty can be reduced. On the other hand, it is usually very difficult to estimate or quantify this uncertainty.

An example of this type of uncertainty can also be found in the strength of the foundation. The information about the foundations may be limited so the parameters used to characterize its resistance are estimated though probing and exploration. With more resources, the foundation can be better characterized and the epistemic uncertainty is reduced, although the natural variability of the foundation may still be very significant.

The distinction between natural and epistemic uncertainty takes added importance for a quantitative risk analysis in complex structures [12]. In this context, natural uncertainty is usually related to the occurrence of events that can produce the structural failure and the randomness of the structure's resistant behavior for the load produced by the events. In contrast, epistemic uncertainty is mainly focused on the lack of knowledge of the loading events, the failure mechanisms, the structure's resistance parameters and the consequences produced by the failure.

Uncertainties in dam safety have been treated in detail by several authors $[13,10,14]$, and discussions include not only parameter and system uncertainty, but also loading uncertainty. Several studies have tried to distinguish 


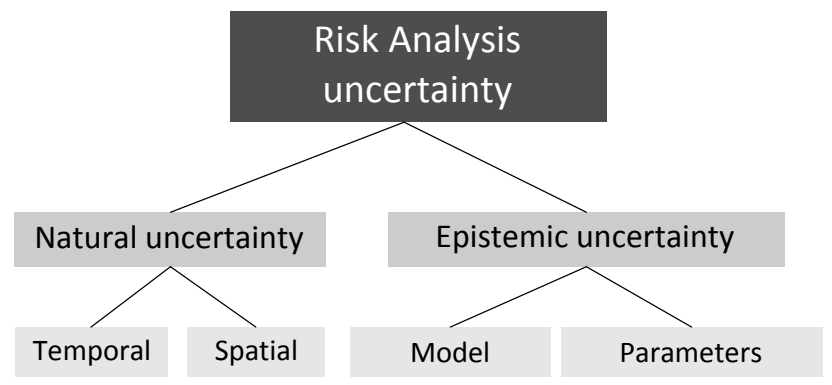

Figure 1: Taxonomy of uncertainty in Risk Analysis (adapted from [10]).

between both types of uncertainty in the dam safety field [15, 16]. In particular, [17] makes a detailed review of epistemic and natural uncertainties for the sliding failure mode of concrete dams.

As explained by Paté-Cornell [18], different levels of risk analysis complexity can be achieved depending on how uncertainty is addressed. In the dam safety field, quantitative risk analysis is commonly addressed defining different failure mechanisms for failure events [4, 19, 20]. In general, a single value of failure probability and risk is estimated for each failure mechanism combining both types of uncertainty.

Other industries like nuclear and aeronautical have achieved a higher level of complexity, with a second-order probabilistic risk analysis based on a full representation and separation of epistemic and natural uncertainty [18]. In this case, a failure probability and risk profile is obtained to represent the influence of epistemic uncertainty in the results. With this approach, the effect of measures for epistemic uncertainty reduction can also be evaluated and compared with risk reduction measures favoring a better informed dam safety management. Altarejos [21] had also suggested a procedure for slopes and embankment dams.

In this paper, the authors present a procedure to adapt the methodology developed in the nuclear industry to the dam safety field. This procedure develops fragility analysis, which accounts for both types of uncertainty. This paper is focused on applying this procedure for the sliding failure mode of concrete gravity dams, although it can be used for fragility analysis of other structural failure modes. The present paper has a broad scope since it is focused on the presented procedure to develop this fragility analysis rather than on reviewing how considering aleatory and epistemic uncertainty in specific parameters and equations of the existing numerical models for the 
sliding failure in concrete dams.

Sliding failure mode has been selected since sliding produced by insufficient shear strength in the foundation is the most common cause of failure of concrete gravity dams according to the International Commission on Large Dams [22]. For this reason, regulatory rules and guidelines in most countries addresses this failure mode and, indeed, it has been recently analyzed with mathematical models and reliability techniques by different authors [23].

The procedure, after being presented, is later is applied to a concrete gravity dam in Spain in order to illustrate how a fragility analysis can be performed and integrated into a risk calculation model to characterize probability of failure and risk in a more comprehensive way.

\section{Fragility analysis and uncertainty}

In the risk analysis context, fragility curves represent a relationship between conditional failure probability and the magnitude of loads that produce failure. Risk is the combination of three concepts: what can happen, how likely is it to happen, and what are its consequences [24]. Following this definition, one possible way to quantify risk is with the following equation [25]:

$$
\text { Risk }=\int P(\text { loads }) \cdot P(\text { response } \mid l o a d s) \cdot C(\text { loads }, \text { response })
$$

Where the integral is defined over all the events under study, $P$ (loads) is the probability of the different load events, $P$ (response|loads) is the conditional probability of the structural response for each load event and $C$ (loads, response) are the consequences of the system response for each load event.

According to this equation, fragility curves address the second term of the equation, providing the conditional failure probability of the structure for a range of loading events. An example for the sliding failure mode of a gravity dam is shown in Figure 2, where the loading state is represented by the water level in the reservoir.

Therefore, fragility curves provide a representation of the uncertainty about the structural response for a load event. Without uncertainty, the structural response (failure or not) for each loading event would be deterministic. 


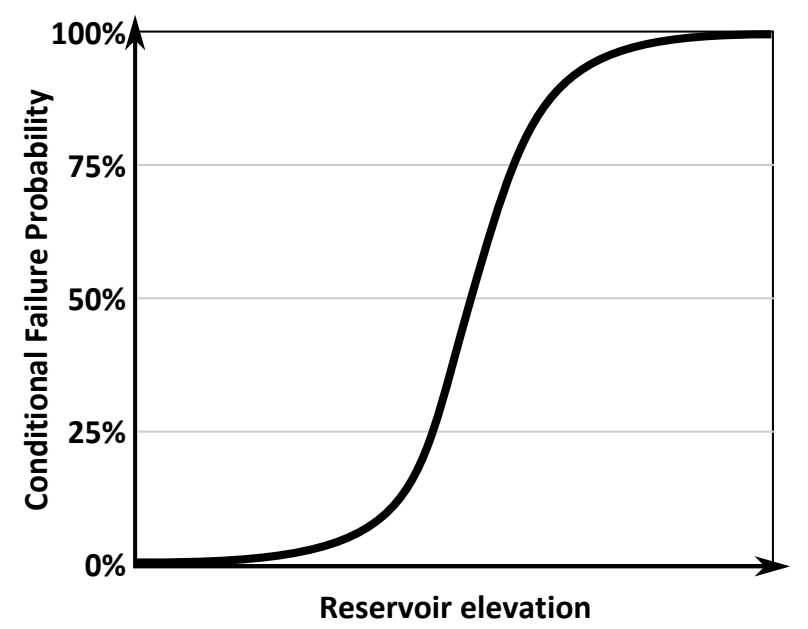

Figure 2: Example of fragility curve for the sliding failure mode.

Different empirical and analytical methodologies have been developed to obtain fragility curves in complex structures [26, 27]. In general, these curves are calculated with reliability analysis techniques, which estimate the probability of the load effect exceeding the resistance effects of the structure. This estimation is made evaluating the uncertainty of the input variables in the structural analysis.

When a single fragility curve is obtained to characterize the system's response, it usually addresses both types of uncertainty: epistemic and natural. Hence, when reliability techniques are applied to obtain a fragility curve, these two types of uncertainty are usually analyzed together in the input variables [13].

In order to separate both types of uncertainty, Kennedy \& Ravindra defined a conceptual framework [28] that was used by EPRI to develop a methodology for risk-informed safety management in the nuclear industry [26]. According to this methodology, the system response for a loading state (i.e. an earthquake event) can be defined by a family of fragility curves instead of a single curve, as shown in Figure 3.

In this methodology, fragility curves are described by log-normal distributions, defined by parameters for the aleatory uncertainty: $m_{R}$ (median or logarithmic mean) and $\beta_{R}$ (logarithmic standard deviation). Epistemic uncertainty is modeled by treating the logarithmic mean as a Bayesian random variable, $M_{R}$, with median $m_{R}$ and log-std dev $\beta_{U}$. Thus, in the family of 


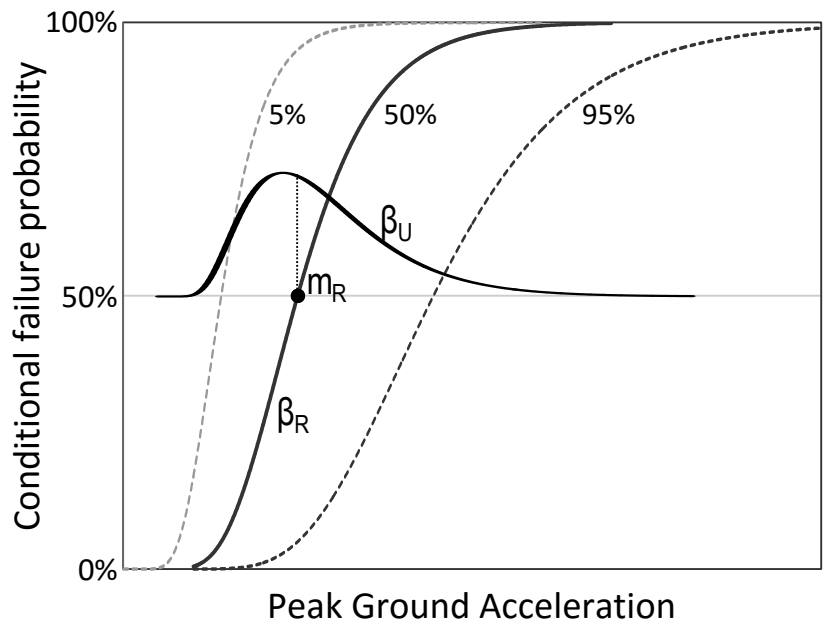

Figure 3: Example of family of seismic fragility curves for a structure. Adapted from [26].

curves defining the fragility, all have the same $\beta_{R}$ and this family characterizes both uncertainties, as shown in Figure 3.

A family of fragility curves provides more information about uncertainty than a single fragility curve that considers natural and epistemic uncertainty combined. Figure Figure 4 idealizes graphically the difference in the resulting fragility curves of these two approaches.

\section{The sliding failure mode in gravity dams}

The sliding failure mode in concrete gravity dams addresses the sliding of the whole dam section or part thereof along the dam-foundation contact and sliding along lift joints in the dam body or along weak planes in the foundation [29]. In fact, analysis of this failure mode is usually a key point of concrete dams' safety reviews. The safety evaluation is typically done using the factor of safety concept, which in general is defined as the ratio between the resistant forces and the driving forces along a giving sliding surface.

In the professional literature, a wide range of deterministic numerical and mathematical models can be found to analyze sliding safety of concrete dams, from 2D limit equilibrium models, which are the most common in international guidelines and regulations, to complex 3D finite element models $[30,31,32,33]$.

In the context of risk and reliability analysis, the most common approach to analyze sliding failure mode is using fragility curves, which combine condi- 


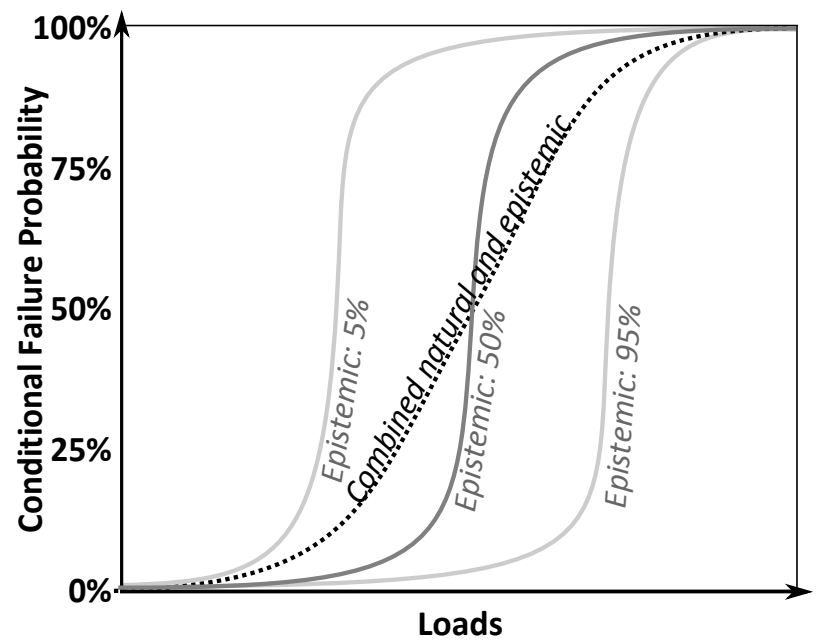

Figure 4: Differences when natural and epistemic uncertainties are separated versus when combined into one only fragility curve.

tional failure probability of the dam for different scenario conditions. These curves represent the system response in the risk model, as explained in the previous section. In general, for a defined loading state, a dam could fail due to different failure modes, controlled by different failure mechanisms. Commonly, fragility curves are defined for each failure mechanism separately and the individual mode fragilities are combined using classical system reliability techniques [4]. Therefore, sliding probability is usually introduced in quantitative risk models through fragility curves, where it is combined with other failure modes to compute overall dam failure probability and risk.

Other approaches followed to address uncertainty in the sliding failure mode has focused on the uncertainty of the hydrological loads and their relation with the system response [34] or on the spatial variation of foundation properties using geostatistical techniques [17].

In the context of risk and reliability analysis, the most common approach to analyze sliding failure mode is analyzed through a conditional failure probability of the dam for different scenario conditions, representing the system response in the risk model, as explained in the previous section.

In the literature, some examples can be found on the calculation of fragility curves for sliding of concrete dams and their use in risk models to compute failure probability $[25,20,35,13,19]$. In these examples, fragility curves are computed combining deterministic numerical models with relia- 
bility techniques. In each numerical model, different random variables are defined and then sampled to compute failure probabilities by analyzing the dam stability for different loading cases. In general, random variables distributions are based on local measurements and in-situ tests of foundation characteristics and drainage system behavior. The random variables defined and the numerical models used are different in each example.

However, these examples all present a significant disadvantage, since natural and epistemic uncertainties are addressed together when obtaining the fragility curves [13]. In fact, in general, a lot of effort has been put into very advanced reliability methods and numerical models, but less effort has been put into the input parameters estimation [20]. Thus, uncertainty in input data can have an overwhelming influence on the resulting failure probability [36].

In this context, addressing epistemic and natural uncertainty separately as proposed in this paper will help identify: 1)the main gaps of knowledge and 2)the effect on the computed failure probability of actions to reduce the epistemic uncertainty (new in-situ/laboratory tests, better monitoring system, analysis with numerical models, etc.).

\section{Procedure for sliding failure fragility analysis}

In this section, a procedure is presented to estimate a family of fragility curves that addresses natural and epistemic uncertainty independently for risk-informed dam safety management. This procedure combines the conceptual framework used in the nuclear industry explained in Section 2 with the existing methods for fragility curves estimation in concrete dams explained in Section 3.

The proposed procedure includes the following steps:

Step 1 Define the mathematical or numerical model that simulates the physical problem. As explained in the previous section, different models can be used to analyze sliding failure, form simple limit equilibrium models to complex non-elastic models [25]. The selection will depend on the complexity of the problem analyzed and the quantity and quality of data available. The performance of the model selected is also a source of epistemic uncertainty that should be analyzed in Step 8.

Step 2 Define the loading range and the number of loading cases analyzed to estimate the fragility curve. For the sliding failure mode, loadings 
are usually represented by the reservoir elevation, since water pressure and uplift are the driving forces for this failure.

Step 3 The working team has to assess which variables of the model will be considered as subjected to none or very low uncertainty, and which variables have necessarily to be treated as random. Typically, for the sliding failure mode, most of the uncertainty lies on the foundation strength and the magnitude of pore water pressures in the foundation soils, including the soil along rock discontinuities, or uplift pressures along such rock discontinuities.

Step 4 Two different distributions should be defined for each random variable: one for natural uncertainty and one for epistemic uncertainty. Mean values, standard deviations and probability distribution should be estimated, based on available data. In order to follow the conceptual framework explained in Section 2, it is proposed that both distributions should have the same mean, while the standard deviations and the probability distribution will depend on the natural and epistemic uncertainty. As explained in Step 7, the distribution defined for the epistemic uncertainty is a distribution of means of the random variables. The use of the mean to define random distributions is recommended, since it represents the expected value of the parameters and and its estimate is relatively stable for small sample sizes, in contrast to the median. Typically used probability distribution, include uniform, normal, log-normal, triangular, and beta distributions.

Distinguishing and defining probabilistic distributions for both types of uncertainty based on tests and measurements data can be difficult and quite subjective. The focus of this paper is not this distinction, which has been widely discussed by several authors [37, 11, 38]. In any case, when these distributions are defined, the working team should keep in mind that the random uncertainty represents the natural variation while the epistemic uncertainty is the part of the uncertainty that cannot be reduced with more test and computations, including the consideration of uncertainty due to not knowing what we do not know.

Step 5 Select the reliability method that will be used in the model to estimate failure probability. Some examples of these methods are First Order Second Moment (FOSM), Taylor's Method, Point Estimate Method 
(PEM), Advanced Second Moment (ASM) Hasofer-Lind Method and Monte Carlo Method [25, 39, 40, 41]. Different reliability methods can be chosen for epistemic and natural uncertainty.

Step 6 For each loading case, compute the conditional failure probability using the selected reliability method for the natural uncertainty. In this step, values of the random variables are selected based on the chosen reliability method (Step 5) and the probability distributions defined for the natural uncertainty (Step 4). Therefore, different computations of the numerical model (Step 1) are made with these selected values to estimate failure probability.

When failure probability is represented versus loading range, the fragility curve capturing natural uncertainty is obtained. This curve is called "reference fragility curve" since it is computed only with the probability distribution for the natural uncertainty, without considering epistemic uncertainty. The "reference fragility curve" is equivalent to the fragility curve that could be obtained following the other existing methods to obtain fragility curves explained in the previous section (if they would only consider the natural uncertainty). Using only this reference fragility curve based on aleatoric uncertainty could underestimate the actual failure probability due to effect of the epistemic uncertainty. This additional uncertainty can be captured in the adjustment proposed in Step 7.

Step 7 First, groups of random variables are selected in the epistemic uncertainty distributions following the reliability method chosen for the epistemic uncertainty.

Second, for each selected group of random variables, a new probability distribution is defined for the natural uncertainty, using as "new mean" these selected values and keeping the same standard deviations and probability distributions defined for the natural uncertainty. Afterwards, for each group of random variables, Step 6 is followed to estimate the corresponding fragility curve using the new probability distribution defined for natural uncertainty. Therefore, the differences between the fragility curves are the mean values of the probability distributions of the random variables used to compute them. As a result, the family of fragility curves obtained separately captures both the epistemic and natural uncertainty. In order to obtain the structure of 
curves and parameters shown in Figure 3, median (logarithmic mean) can be obtained based on these results.

Step 8 Check the outcomes and perform sensitivity analysis on any of the decisions previously taken. This last step is a crucial, as the engineer should never get lost in any mathematical approach that may not represent sound engineering judgment.

The same structure followed in this method can be applied to other failure modes and dams' typologies to obtain fragility curves. Particularly for those failure modes of essentially "structural" nature, this method can be carried out rather straightforwardly due to its generic formulation.

Finally, this family of fragility curves can be introduced into a complete and quantitative risk model to estimate failure probability and risk, analyzing the effect of reducing epistemic uncertainty in the risk results.

\section{Case study: Spanish concrete dam}

The procedure described in the previous section is applied here to estimate a family of fragility curves for the sliding failure mode in a Spanish concrete dam. In this case, sliding along the concrete-foundation interface was considered for the central section. This section presents a triangular profile, as shown in Figure 5, with slopes of 0.05/0.76 (Upstream/Downstream). The dam crest level is at +911.4 meters and the foundation level is at +915.2 meters The height of the cross section is $96.2 \mathrm{~m}$ above the foundation. The maximum operating level in the reservoir is +908.5 meters. The dam is located over a sub-vertical layer of quartzite of $70 \mathrm{~m}$ depth. These quartzites have cracked and split into fragments due to the effect of its fold. Vertical cracks are predominant due to the strong vertical folding.

According to previous analyses and studies, the engineers in charge of this dam had doubts about the safety of this dam for the sliding failure mode. For this reason, risk analysis was applied to estimate the current risk for the sliding failure mode in the dam and to propose potential risk reduction measures and/or measures to reduce epistemic uncertainty. Epistemic uncertainty was significant in this case, due to the lack of data about the original design and the foundation characteristics, so it was considered that separating both types of uncertainty was important for a proper risk-informed dam safety management. 


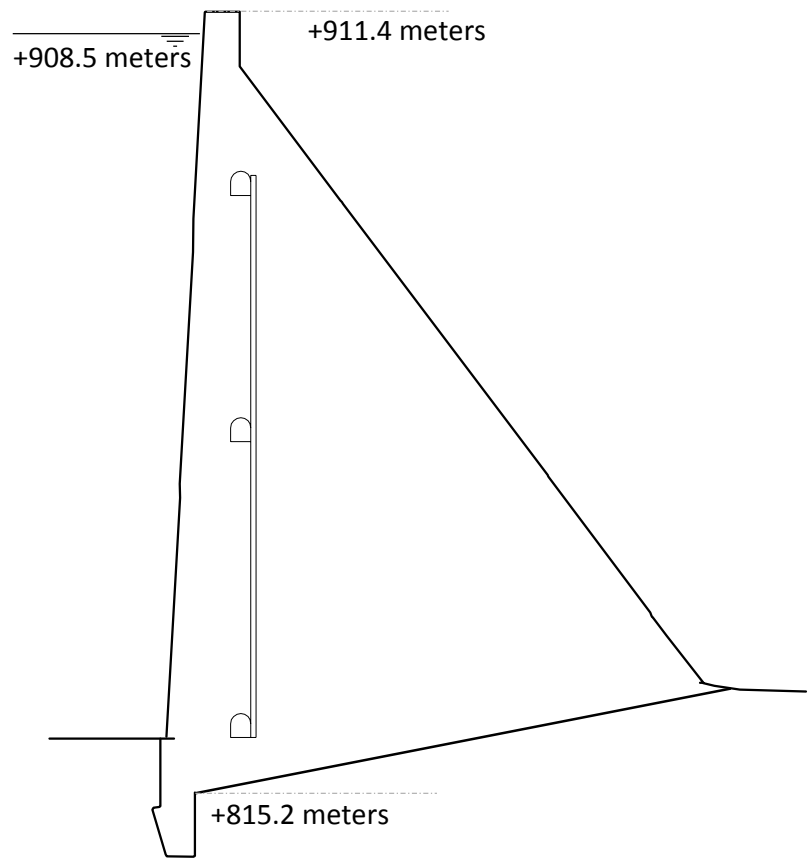

Figure 5: Cross section of the case study concrete dam. 
Next, the steps presented in the previous section are followed to estimate the family of fragility curves and the risk profile in the dam, making a significant number of decisions that are justified as suitable for this particular problem.

Step 1. Identify the mathematical o numerical problem that represents the sliding failure mode. In this case, a 2D Limit Equilibrium Model was used to evaluate sliding failure along the foundation-concrete interface. The model includes a single interface in the contact between the dam and the foundation. This interface can mobilize tensile strength up to some limit value. The model allows for crack opening and propagation, with full uplift under the cracked zone of the dam base.

The limit-state function is defined as the ratio between the resistant force and the driving forces. In the cases where the driving forces are higher that the resistant forces, is considered that the dam would fail. The resistant force is supposed to be controlled exclusively by the friction angle and cohesion at the dam-foundation contact, following the classical Mohr-Coloumb equation:

$$
R=(N-U) \cdot \tan (\varphi)+B \cdot c
$$

Where $N$ are the normal forces acting on the contact $(k N / m), U$ the uplift force $(k N / m), \varphi$ is the friction angle $\left(^{\circ}\right), B$ is the compressed length on the contact $(m)$, and $c$ is the cohesion $\left(k N / m^{2}\right)$.

The driving forces are the reservoir water pressure, the submerged sediments pressure and the uplift pressure. Water and uplift pressures directly depend on the water level in the reservoir.

Step 2. Define the plausible loading range that can produce the sliding failure. The selected variable to define the loading state in the dam is the maximum water level reached in the reservoir during any given hydrologic event, since water pressure is the main driving force of the sliding failure mode.

The selected range of pool levels comprises from +908 meters $(0.5$ meters under the maximum operating level) to +915.4 meters ( 4 meters above the dam crest level, for this level the overtopping failure mode would be clearly predominant). In total, 38 reservoir elevations (equally distributed every 0.2 meters within the range) were considered for calculations.

Step 3. Define which variables of the mathematical model will be treated as random. In this case, since the uncertainty on the foundation resistance capacity is the main concern, two independent random variables of the Limit 
Equilibrium Model are considered based on an initial sensitivity analysis: friction angle $(\varphi)$ and cohesion $(c)$.

All the other variables are assumed to be known exactly, including geometrical parameters such as dam height, upstream slope, downstream slope, etc., material characteristics, such as concrete density, and performance parameters such as drain effectiveness (which considered as completely ineffective).

Step 4. Estimate two statistical distributions (for natural and epistemic uncertainty) for each of the random variables. Two probabilistic distributions have been estimated for both random variables as shown in Table 1. The estimation of these probabilistic distributions by expert judgement is a complex process which is out of the scope of this paper and it should be based on geotechnical in-situ and laboratory tests, measurements procedures and temporal and spatial variations of results. As can be observed, the same means are used for both types of uncertainty as stated in the previous section. The working team has evaluated which part of the uncertainty is due to natural variations in the foundation and which part could be reduced with more tests and data. The standard deviation has been considered higher for the epistemic uncertainty than for the natural uncertainty, due to the existing doubts about the dam foundation. The truncated normal distribution is used for the friction angle and the truncated lognormal distribution is used for the cohesion, as these are the distributions that best fit available data. This result is similar to others found in literature [42, 33, 43].

\begin{tabular}{cccccc}
\hline Random variable & Mean & St. Deviation & Maximum & Minimum & Type \\
\hline \multicolumn{5}{c}{ Natural uncertainty } \\
\hline $\begin{array}{c}\text { Friction angle } \\
\text { Cohesion }\end{array}$ & $50^{\circ}$ & $5^{\circ}$ & $65^{\circ}$ & $35^{\circ}$ & Normal \\
\hline \multicolumn{7}{c}{$\mathrm{MPa}$} & $0.2 \mathrm{MPa}$ & $1.5 \mathrm{MPa}$ & $0.1 \mathrm{MPa}$ & Log-Normal \\
\hline $\begin{array}{c}\text { Mean friction angle } \\
\text { Mean cohesion }\end{array}$ & $5.5 \mathrm{MPa}$ & $0.125 \mathrm{MPa}$ & $1 \mathrm{MPa}$ & $0.2 \mathrm{MPa}$ & Log-Normal \\
\hline
\end{tabular}

Table 1: Estimated probability distributions for the random variables.

Step 5. Select the reliability method to estimate failure probability. The reliability method selected for both natural and epistemic uncertainty is the Monte Carlo with the Latin Hypercube Sampling (LHS) method. This method is chosen because the state function and calculation model selected 
in Step 1 are simple enough to allow many limit equilibrium computations to be rapidly made. Furthermore, for both types of uncertainty, 10,000 samples of random variables are made to define the family of fragility curves.

Step 6. Build the reference fragility curve. First, 10,000 pairs of values of the random variables $(\varphi, c)$ have been sampled with the Latin Hypercube method in the probabilistic distributions for the natural uncertainty.

Second, for each water level, the Limit Equilibrium Model is used to define the limit curve for these two random variables [25]. The limit curve represents the values of the random variables $(\varphi, c)$ that produced a resistance force equal to the driving forces. Conditional failure probability is estimated with the number of sampled values of the random variables that are located under the limit curve (failure cases). For example, this process is represented in Figure 6 for one water level (among the 38 water levels considered).

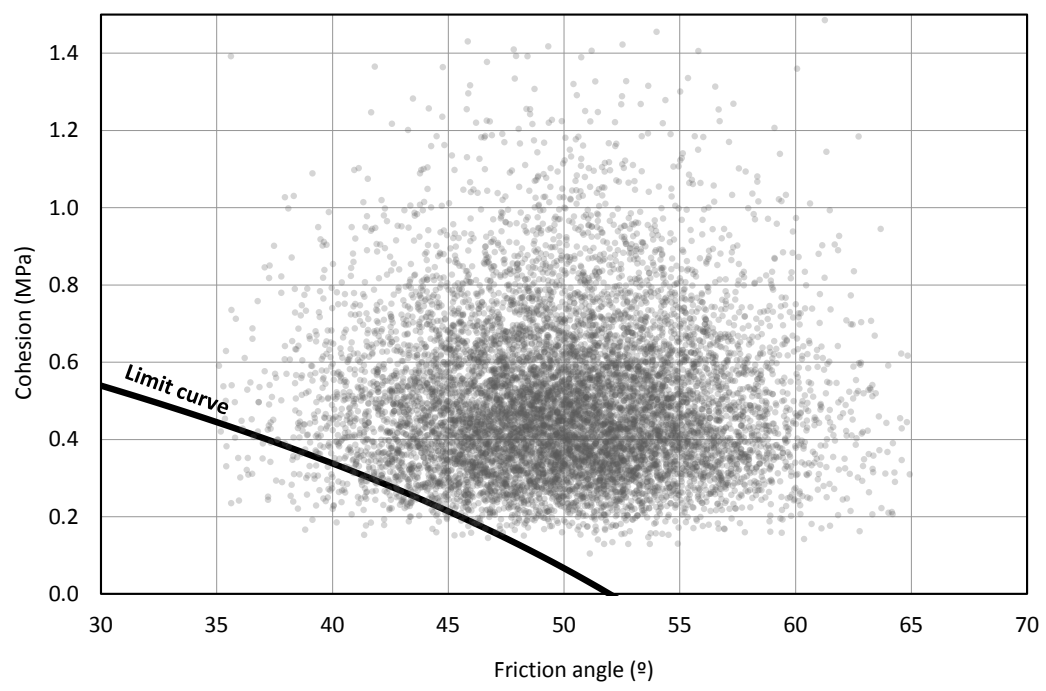

Figure 6: Limit curve for pool water level 911.4 meters (Conditional failure probability = $1.75 \%)$.

When conditional failure probability is represented versus pool water level, the reference fragility curve is obtained. This curve only reflects aleatory uncertainty and it is shown in Figure 7. The obtained curve shows that, according to the chosen mathematical model, the dam has a quite fragile response for the sliding failure mode, since the curve changes from $0 \%$ to $100 \%$ conditional failure probability with a difference of 4 meters in the pool 
water level.

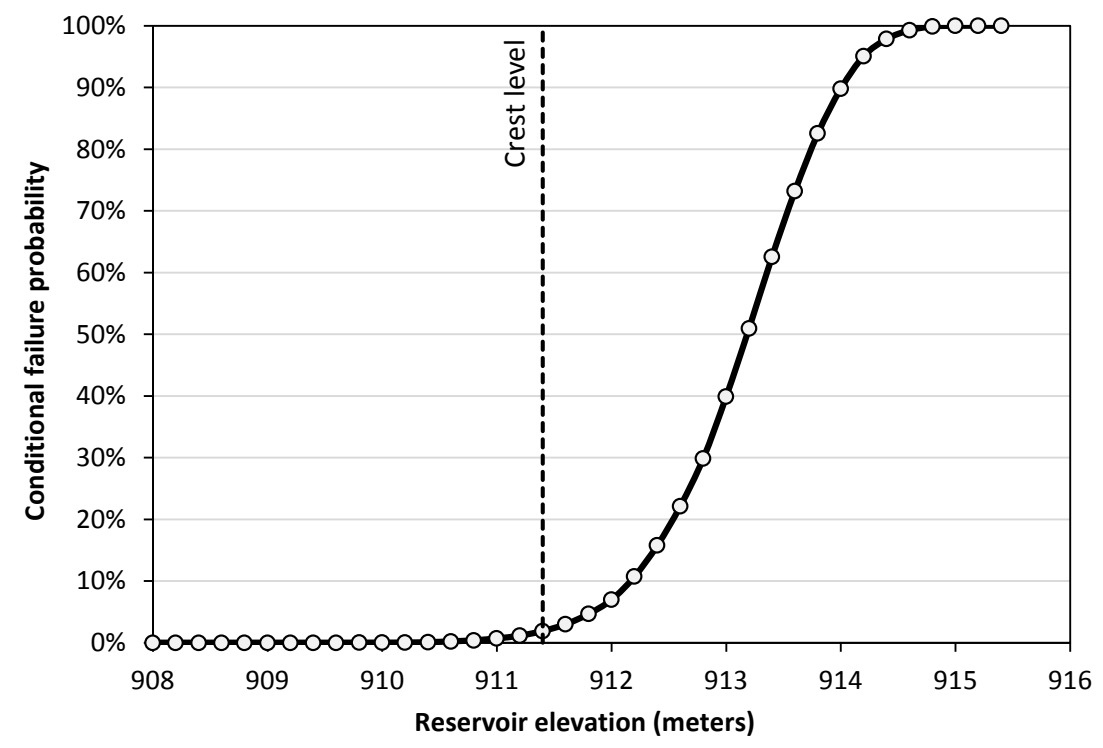

Figure 7: Reference fragility curve for the case study.

Step 7. Build the family of fragility curves combining the probability distributions defined for epistemic and natural uncertainties. In this step, a family of 10,000 fragility curves is obtained to characterize epistemic uncertainty. First, 10,000 couples of values of the random variables $(\varphi, c)$ have been sampled with the LHS method in the probabilistic distributions for the epistemic uncertainty.

Second, each couple of values has been used to define two new probabilistic distributions for $\varphi$ and $c$. In these distributions, the sampled values are the mean, while the type of distribution and the standard deviation are the same that in the natural uncertainty probabilistic distribution.

Third, for each couple of values, Step 6 is applied to obtain a fragility curve using the new probabilistic distributions for $\varphi$ and $c$. Therefore, 10,000 fragility curves are obtained, one for each pair of values. In order to obtain all these fragility curves, the dam stability is checked 38 (number of water levels) x 10,000 (number of curves) x 10,000 (number of sampled values to estimate failure probability) $=3.8$ billion times. The family of fragility curves obtained is shown in Figure 8.

Figure 8 provides very useful information for dam safety governance and decision making. For instance, it indicates that at the current crest elevation 


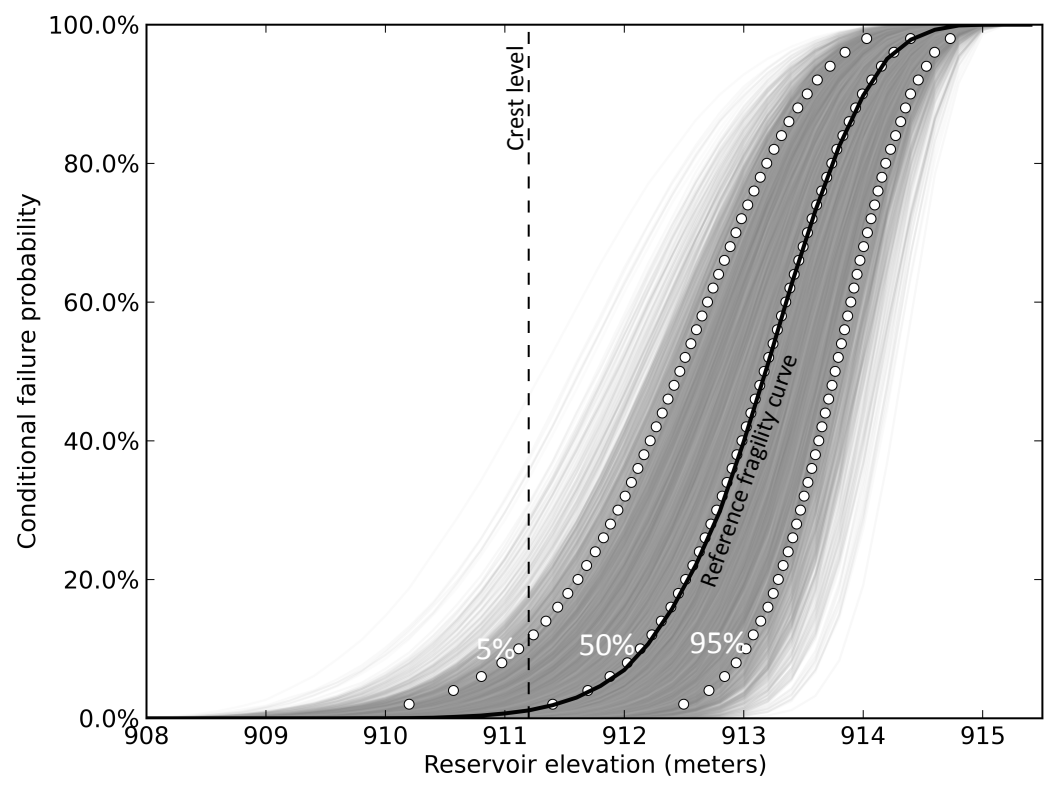

Figure 8: Family of fragility curves obtained for the case study.

of 911.5 meters, there is a level of confidence higher than $95 \%$ that the dam will not fail by sliding, since probability of failure for the $5 \%$ curve is lower than $20 \%$. In addition, probability of sliding failure is very low for reservoir elevation lower than 909 meters.

As can be observed in this figure, these fragility curves are not parallel, since there are two independent random variables. The spread of this family is an indicator of the influence of the epistemic uncertainty in the results. Furthermore, in order to follow the EPRI framework (Section 2), the standard deviation $\beta_{U}$ can be obtained representing the histogram of the median of the curves (conditional probability $=50 \%$ ), as shown in Figure 9. In this case, the standard deviation of this probability distribution is 0.40 meters and the median +913.17 meters.

Step 8. Check results and re-evaluate previous decisions. In this step, the different decisions made in the previous steps should be reviewed and the obtained results should be checked. The simple Limit Equilibrium Model selected for this analysis has allowed completing a high number of stability computations in order to explain the proposed procedure in detail. Although, this simple model is also a source of epistemic uncertainty that could be 


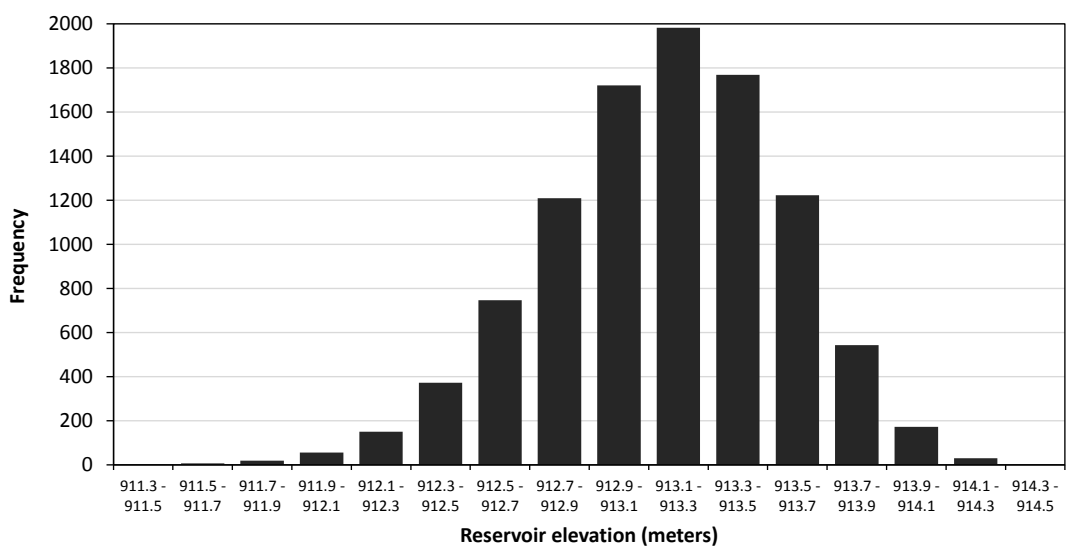

Figure 9: Histogram of the median in the 10,000 fragility curves.

evaluated comparing it with more complex stability models.

One of the issues that has a higher influence in the results and the calculating times is the number of samples of the random variables chosen to estimate failure probability. In order to analyze the effect of this decision, the failure probability within the reference fragility curve has been re-estimated depending on the number of samples for three different maximum water levels: $+912,+913$ and +914 meters Results are shown in Figure 10 .

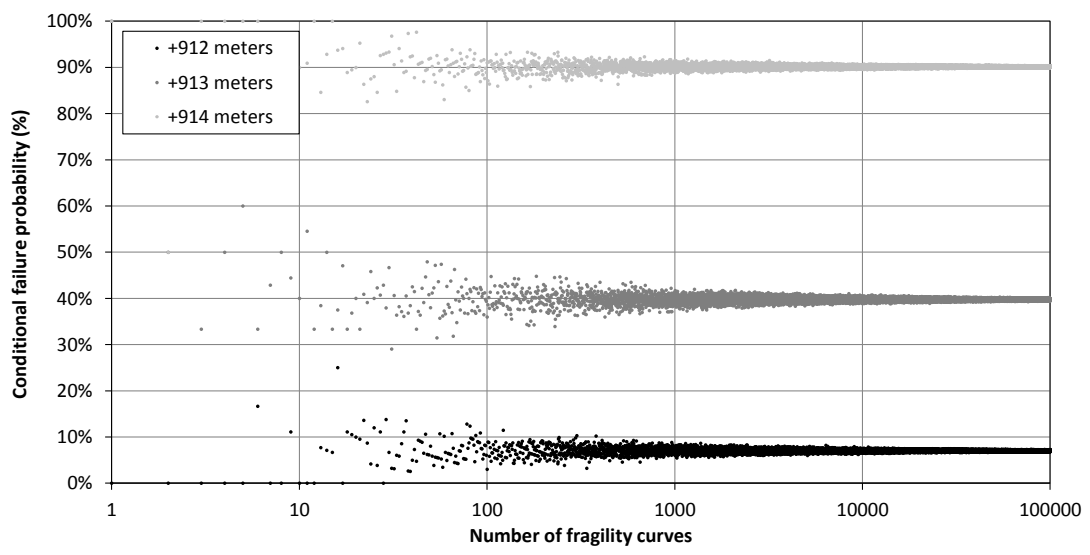

Figure 10: Failure probability for three reservoir elevations as a function of the number of samples used to compute them.

As can be perceived in Figure 10, failure probability results are stable 
when the number of samples is higher than 1,000, so 10,000 samples is a good decision to achieve accurate results.

Next, the number of fragility curves needed to properly characterize epistemic uncertainty is addressed. In this case, the standard deviation of the histogram of the fragility curves family has been estimated as a function of the number of fragility curves used to compute it for three different conditional failure probabilities $(5 \%, 50 \%$ and $95 \%)$. This standard deviation is an indicator of the spread of the family of fragility curves.

As can be observed in Figure 11, standard deviation results are stable when the number of fragility curves is higher than 1,000. Therefore, 10,000 fragility curves seems a good decision.

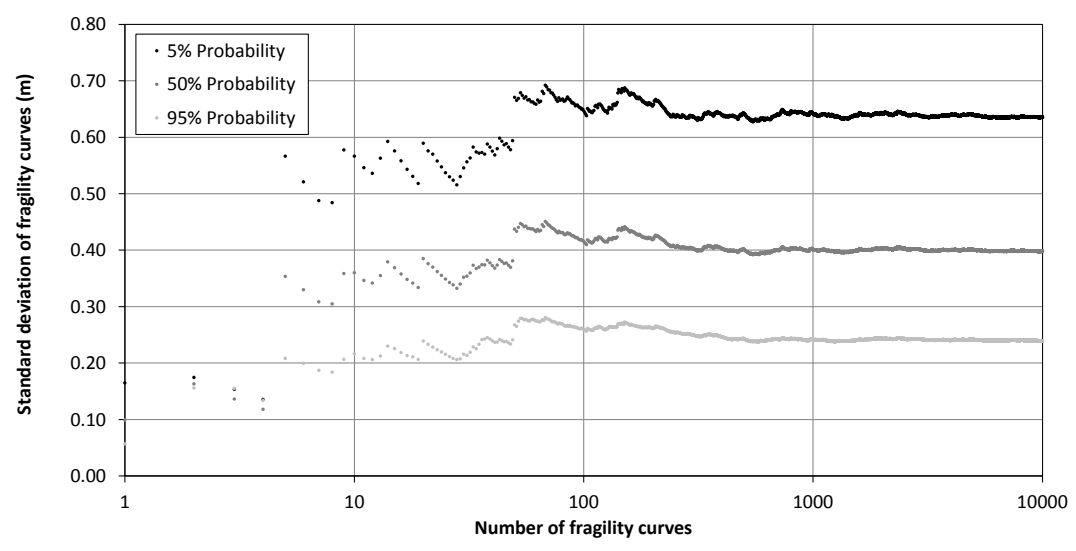

Figure 11: Standard deviation of the histogram of the fragility curves family as a function of the number of fragility curves considered.

\section{Discussion and final remarks}

The paper formulates a comprehensive procedure for fragility analysis of sliding failure of concrete gravity dams making use of reliability methods and formally distinguishing two main sources of uncertainty, natural and epistemic. The suggested eight step framework focuses on the options and decisions that an analyst has to face, trying not to hide crude real world decisions behind the elegance and accuracy of mathematics. As a matter of fact, fundamental issues remain to some extent unsolved and it is not our intention to avoid the debate, on the contrary, we believe that any innovation or advance in analysis techniques should facilitate it. Some conceptual and operational precautions have been observed. Namely: 
- Friction and cohesion (in the case study presented in this paper) but also other variables potentially representing "relevant data" to other cases, such as uplift or ice load pressure, are quite difficult to fully characterize by means of empirical data, regardless the number of data available, due to facts such as imperfect testing and/or non fully reliable measuring techniques among other reasons. Indeed, the independence hypothesis among them is not universally agreed, or strongly contested.

- The impact on the results of the choice of the probability distributions has been addressed. Defining separated probability distributions for natural and epistemic uncertainty is a complex process that should be based on tests data and expected model and measurements errors.

- This framework is focused on the system response term of the risk equation, but distinguishing natural and epistemic uncertainty is also necessary when loads and consequences are addressed. Therefore, uncertainties can be propagated in the risk equation through convolution.

- Nevertheless, the more the procedure decomposes the process, and the more decisions and options are subjected to open discussion and scrutiny, as we think our procedure does, we truly believe that results are more meaningful for understanding the risks and informing decision making.

On the other hand, the separation of epistemic uncertainty and natural variability following the proposed methodology is not only an academic exercise, but strong practical implications can be followed from this separation in the everyday safety management decisions. It is worth considering that:

- The impact of the epistemic uncertainty span on the overall risk picture appears as valuable information regarding the design and urgency of the actions needed to reduce the gap in knowledge and so to improve the safety of the dam.

- The decision-maker is provided with information of the estimated residual risk level expected after gaps in knowledge have been filled. By deriving a probability function estimate for natural variability, the working team is expressing a minimum value regarding how much uncertainty is going to be assumed by the owner on the current situation, 
given that the gaps in knowledge are filled, without further risk reduction measures.

- This residual risk picture generated by natural variability can be used to inform the design of additional risk reduction measures aimed to reduce the loading probability or to reduce the failure probability.

- In addition, having adopted the EPRI distinction between epistemic and natural variability, brings together the practices of two different industries, nuclear and dams, favoring a needed dialogue for performing analysis such as the nuclear risk assessment for facilities that can potentially be flooded by failure of upstream dams.

Finally, it is worth to mention that the intent of the authors is coping with uncertainty, more than fighting against uncertainty, which is identified as one of the main elements of "smart governance" [44].

\section{Acknowledgements}

This paper was published with the support of the research project "INICIA" (Methodology for Assessing Investments on Water Cycle Infrastructures informed on Risk and Energy Efficiency Indicators, BIA2013-48157C2-1-R, 2014-2016), co-funded by the Spanish Ministry of Economy and Competitiveness "Ministerio de Economía y Competitividad" (Programa Estatal de Investigación, Desarrollo e Innovación Orientada a los Retos de la Sociedad) and the European Regional Development Fund (ERDF).

\section{References}

[1] G. Augusti, M. Ciampoli, Performance-based design in risk assessment and reduction, Probabilistic Engineering Mechanics 23 (2008) 496-508.

[2] EC, Council directive 82/510/ec of 24 june 1982 on the control of majoraccident hazards of certain industrial activities, European Comission (1982).

[3] USNRC, White paper on risk-informed and performance-based regulation, United States Nuclear Regulatory Commission, 1999. 
[4] SPANCOLD, Risk analysis applied to management of dam safety - Technical Guide 8 (Vol. 1), Spanish National Committee on Large Dams, 2012.

[5] K. Petersen, European model evaluation activity, Journal of Loss Prevention in the Process Industries 7 (2).

[6] D. Veneziano, A. Agarwal, E. Karaca, Decision making with epistemic uncertainty under safety constraints: An application to seismic design, Probabilistic Engineering Mechanics 24 (2009) 426-437.

[7] F. Silva, T. Lambe, W. Marr, Probability and risk of slope failure, Journal of geotechnical and geoenvironmental engineering (2008) 1691-1699.

[8] F. O. Hoffman, J. S. Hammonds, Propagation of uncertainty in risk assessments: The need to distinguish between uncertainty due to lack of knowledge and uncertainty due to variability, Risk Analysis 14 (5) (1994) 707-712.

[9] S. Ferson, L. Ginzburg, Different methods are needed to propagate ignorance and variability, Reliability Engineering and System Safety 54 (1996) 133-144.

[10] D. Hartford, G. Baecher, Risk and uncertainty in dam safety, Thomas Telford Ltd. CEA Technologies. Dam Safety Interest Group, 2004.

[11] A. Der Kiureghian, O. Ditlevsen, Aleatory or epistemic? does it matter?, Structural safety 31 (2009) 105-112.

[12] P. Baraldi, E. Zio, A combined Monte Carlo and possibilistic approach to uncertainty propagation in event tree analysis, Risk Analysis 28 (5) (2008) 1309-1325.

[13] B. Ellingwood, P. Tekie, Fragility analysis of concrete gravity dams, Journal of infrastructure systems 7 (2) (2001) 41-48.

[14] H. Kreuzer, Assessing uncertainty in dam engineering, in: 73rd Annual Meeting of ICOLD. Paper 007-S1. Teheran, Iran., 2005.

[15] C. Carvajal, L. Peyras, C. Bacconnet, J-P. Bécue, Probability Modelling of Shear Strength Parameters of RCC Gravity Dams for Reliability, 
European Journal of Environmental and Civil Engineering 13 (1) (2009) 91-119.

[16] C. Carvajal, L. Peyras, C. Bacconnet, On the loading/shear strength coupling in the probabilistic formulation of the limit-state in shear for gravity dams, European Journal of Environmental and Civil Engineering 14 (3) (2010) 283-301.

[17] L. Altarejos-García, I. Escuder-Bueno, A. Morales-Torres, Advances on the Failure Analysis of the Dam-Foundation Interface of Concrete Dams, Materials 8, (2015) 8255-8278.

[18] E. Paté-Cornell, Risk and uncertainty analysis in government safety, Risk Analysis 22 (3) (2002) 633-646.

[19] L. Peyras, C. Carvajal, H. Felix, C. Bacconnet, Royet, P., D. B. J.P., Boissier, Probability-based assessment of dam safety using combined risk analysis and reliability methods - application to hazards studies, European Journal of Environmental and Civil Engineering 16 (7) (2012) 795-817.

[20] M. Westberg, Reliability-based assessment of concrete dam stability, Doctoral Thesis. Lund University. Division of Structural Engineering, Lund (Sweden), 2010.

[21] L. Altarejos-García, F. Silva-Tulla, I. Escuder-Bueno, A. MoralesTorres, Practical risk assessment for embankments, dams, and slopes. Risk and Reliability in Geotechnical Engineering, CRC Press, 2015, Ch. 11, pp. 437-470.

[22] ICOLD, Dam failures - statistical analysis, Bulletin 99. International Commission on Large Dams, 1995.

[23] H. Kreuzer, The adjustable factor of safety, in: Hydropower and Dams, 2012.

[24] S. Kaplan, The words of risk analysis, Risk Analysis 17 (4) (1997) 407-417. 
[25] L. Altarejos-García, I. Escuder-Bueno, A. Serrano-Lombillo, M. de Membrillera-Ortuño, Methodology for estimating the probability of failure by sliding in concrete, Structural Safety 36-37 (2012) $1-13$.

[26] EPRI, Methodology for Developing Seismic Fragilities, Electric Power Research Institute, 1994.

[27] M. Shinozuka, M. Q. Feng, J. Lee, T. Naganuma, Statistical analysis of fragility curves, Journal of Engineering Mechanics 126 (2000) 1224-1231.

[28] R.P. Kennedy, M.K. Ravindra, Seismic fragilities for nuclear power plant risk studies, Nuclear Engineering and Design 79 (1) (1984) 47-68.

[29] H. Link, The sliding stability of dams, Water Power 21 (1969) 135-139.

[30] L. Altarejos-García, Contribution to the estimation of the probability of failure of concrete gravity dams in the risk analysis context, Doctoral thesis. Universitat Politècnica de València, Valencia (Spain), 2009.

[31] M. Azmi, P. Paultre, Three-dimensional analysis of concrete dams including contraction joint non-linearity, Engineering Structures 24 (2002) $757-771$.

[32] Q. Ren, L. Xu, Y. Wan, Research advance in safety analysis methods for high concrete dam, Science in China Series E: Technological Sciences 50 (1) (2007) 62-78.

[33] G. Ruggeri, R. Pellegrini, M. R. de Célix, M. Bernsten, P. Royet, V. Bettzieche, W. Amberg, A. Gustaffsson, T. Morison, G. Zenz, Sliding safety of existing gravity dams. final report. (2004).

[34] C. Carvajal, L. Peyras, P. Arnaud, D. Boissier, P. Royet, Probabilistic Modelling of Flood Water Level for Dam Reservoirs, ASCE Journal of Hydrologic Engineering, 14 (3) (2009) 223-232.

[35] A. Lupoi, C. Callari, A probabilistic method for the seismic assessment of existing concrete gravity dams, Structure and Infrastructure Engineering: Maintenance, Management, Life-Cycle Design and Performance $8(10)$. 
[36] K. Bury, H. Kreuzer, Assessing the failure probability of gravity dams, Water Power \& Dam Construction.

[37] M. Faber, On the treatment of uncertainties and probabilities in engineering decision analysis, Journal of Offshore Mechanics and Arctic Engineering 127 (3) (2005) 243-248.

[38] A. Jones, S. Kramer, P. Arduino, Estimation of Uncertainty in Geotechnical Properties for Performance-Based Earthquake Engineering, Tech. rep., Pacific Earthquake Engineering Research Center (2002).

[39] C. Cornell, First order uncertainty analysis of soils deformation and stability, in: Proceedings 1st Int. Conf. on application of probability and statistics in soil and structural engineering. Hong Kong, 1971.

[40] E. Rosenblueth, Two-point estimates in probabilities, Applied Mathematical Modelling 5 (5) (1981) 329-335.

[41] A. Hasofer, L. N.C., Exact and invariant second moment code format, Journal of the Engineering Mechanics Division 100 (1) (1974) 111-121.

[42] A. Husein, W. Hassan, A. F.A., Uncertainty and reliability analysis applied to slope stability, Structural Safety 22 (2) (2000) 161-187.

[43] G. Fenton, D. Griffiths, M. Williams, Reliability of traditional retaining wall design, Géotechnique 55 (1) (2005) 55-62.

[44] H. Willke, Smart Governance: Governing the Global Knowledge Society, Campus Verlag, 2007. 